OPEN ACCESS

Edited by:

Tatsuya Sasaki,

F-Power Inc., Japan

Reviewed by:

Vasileios Basios,

Université Libre de Bruxelles, Belgium

Marco G. Mazza

Max Planck Institute for Dynamics and Self Organization (MPG), Germany

${ }^{*}$ Correspondence:

Victor M. Eguíluz victor@ifisc.uib-csic.es

Specialty section:

This article was submitted to Interdisciplinary Physics, a section of the journal

Frontiers in Physics

Received: 13 October 2017 Accepted: 16 February 2018 Published: 07 March 2018

Citation:

Tuzón P, Fernández-Gracia J and Eguiluz VM (2018) From Continuous to Discontinuous Transitions in Social

Diffusion. Front. Phys. 6:21.

doi: 10.3389/fphy.2018.00021

\section{From Continuous to Discontinuous Transitions in Social Diffusion}

\author{
Paula Tuzón ${ }^{1}$, Juan Fernández-Gracia ${ }^{2}$ and Víctor M. Eguiluz ${ }^{2 *}$ \\ ${ }^{1}$ Departament de Didàctica de les Ciències Experimentals i Socials, Facultat de Magisteri, Universitat de València, València, \\ Spain, ${ }^{2}$ Instituto de Física Interdisciplinar y Sistemas Complejos (CSIC-UIB), Palma de Mallorca, Spain
}

Models of social diffusion reflect processes of how new products, ideas, or behaviors are adopted in a population. These models typically lead to a continuous or a discontinuous phase transition of the number of adopters as a function of a control parameter. We explore a simple model of social adoption where the agents can be in two states, either adopters or non-adopters, and can switch between these two states interacting with other agents through a network. The probability of an agent to switch from non-adopter to adopter depends on the number of adopters in her network neighborhood, the adoption threshold $T$ and the adoption coefficient a, two parameters defining a Hill function. In contrast, the transition from adopter to non-adopter is spontaneous at a certain rate $\mu$. In a mean-field approach, we derive the governing ordinary differential equations and show that the nature of the transition between the global non-adoption and global adoption regimes depends mostly on the balance between the probability to adopt with one and two adopters. The transition changes from continuous, via a transcritical bifurcation, to discontinuous, via a combination of a saddle-node and a transcritical bifurcation, through a supercritical pitchfork bifurcation. We characterize the full parameter space. Finally, we compare our analytical results with Monte Carlo simulations on annealed and quenched degree regular networks, showing a better agreement for the annealed case. Our results show how a simple model is able to capture two seemingly very different types of transitions, i.e., continuous and discontinuous and thus unifies underlying dynamics for different systems. Furthermore, the form of the adoption probability used here is based on empirical measurements.

\section{Keywords: adoption, phase transition, mean-field, social contagion, spreading}

\section{INTRODUCTION}

Spreading processes are ubiquitous in nature: the contagion of diseases [1], herd behavior in animals [2], the diffusion of innovations [3], rumor spreading [4], the evolution of social movements [5], the propagation of hashtags in Twitter [6], etc. All these processes share similar dynamics; in a population of initially neutral (disease-free, unaware of some information, etc.) agents (humans, animals, or even bots), some of them start carrying some information, pathogen, or behavior, i.e., they adopt this innovation. Through a transmission process they can pass it on to other agents, starting in this way the process of adoption diffusion.

The diffusion of adoption has been extensively studied and modeled in several fields including Biology, Physics and Social Sciences [7-10]. In general, new adopters have been in contact with 
one or several adopters, with two main mechanisms: in diseaselike models [11, 12], adoption takes place with an adoption probability per contact with an adopter which is constant irrespective of the number of adopters; in threshold-like models [8,11-13], adoption happens only after a critical number of adopters has been reached. There are also models of "generalized contagion" [14], where both disease-like and threshold behaviors are special cases.

However, while the models describe individual adoption probabilities, most of the related empirical research was based on aggregated data, typically cumulative adoption curves $[15,16]$. Recent studies have focused on individuals' behavior, where the number of adopters accessed by each individual can be measured [17-20]. These measurements have a direct connection with the form of the adoption probability. In this paper we explore the probability function obtained by Milgram et al. [17] from a social experiment. They analyzed the correlation between the size of a group looking at the same point in the street and the number of passerby that joined the behavior of looking at that point. The results of the experiment can be fitted with a Hill function for the probability of adoption [20]. We will show that the shape of the adoption probability leads to two different behaviors depending on the parameter values: either a continuous or a discontinuous phase transition. This provides a simple model that describes both regimes within the same framework, depending only on two parameters; with a probability function linked to empirical data.

\section{RESULTS}

An agent that has not adopted yet, adopts with some probability when interacting with an adopter, which turns her an adoptermaker too. After adoption, the agent is "recovered" at a certain rate $\mu$ and becomes again a potential adopter. Here, we study the consequences of the probability of adoption. The transition from adopter to non-adopter is assumed to occur at some constant rate $\mu$.

In the standard SIS (susceptible-infected-susceptible) model [1], the adoption probability (from susceptible to infected, $S \rightarrow$ I) $\beta$ is constant for each interaction with an adopter. In general, the adoption probability can be a general function of the number of adopted neighbors, $n$ :

$$
P(n)=\lambda^{\prime} f(n) .
$$

In this contribution we will consider the function proposed by Gallup et al. [20]

$$
f(n)=\frac{n^{a}}{T^{a}+n^{a}},
$$

where $\lambda^{\prime}$ is persuasion capacity (similar to $\beta=\lambda^{\prime}$ for $T=0$ and $a=1$ ), $a$ is the adoption coefficient (or Hill coefficient) and controls how fast/slow this probability increases with $n$ and $T$ is the adoption threshold and fixes the number of adopters needed to reach half the persuasion limit. $\lambda^{\prime}, T$ and $a$ are real positive numbers. This type of function is known as Hill function and has been used in models of population growth and decline [2123]. The evolution of such a system in an annealed degree regular network (a network where all the nodes have the same number of neighbors or degree $k$ but where they are chosen randomly in the population at each interaction) is determined by

$$
\frac{d \rho}{d t^{\prime}}=-\mu \rho+(1-\rho) A,
$$

where $\rho$ is the density of adopters and $A$ is the probability of adoption given the density $\rho$ and is given by

$$
A=\sum_{n=0}^{k} P(n)\left(\begin{array}{l}
k \\
n
\end{array}\right) \rho^{n}(1-\rho)^{k-n} .
$$

The number of infected neighbors is assumed to be binomially distributed with a success probability equal to the global density of infected agents. Without loss of generality we get rid of parameter $\mu$ by changing the timescale and rescaling the persuasion capacity $\lambda^{\prime}$

$$
\begin{aligned}
t & =\mu t^{\prime} \\
\lambda & =\frac{\lambda^{\prime}}{\mu},
\end{aligned}
$$

which is equivalent to setting $\mu=1$. The equilibrium solutions for the system are determined by the condition

$$
-\rho^{*}+\left(1-\rho^{*}\right) A^{*}=0 .
$$

Given a particular value of $a$ and $T$, there are at most three possible solutions for $\rho^{*}$ (Figure 1): (i) $\rho^{*}=0$, corresponding to the adoption-free regime, (ii) $\rho^{*}=\rho^{u p}$, represented by the upper branch, and (iii) $\rho^{*}=\rho^{\text {down}}$, the lower branch.

The stability of the fixed points can be easily checked by linear stability analysis. The solution $\rho^{*}=0$ changes stability at

$$
\lambda_{0}=\frac{1}{k f(1)}
$$

being stable for $\lambda<\lambda_{0}$ and unstable otherwise. As can be seen in Figure 1, if the solution $\rho^{*}=0$ intersects the upper branch, then that branch is stable and the solution $\rho^{*}=0$ changes stability via a transcritical bifurcation. Then for $\lambda>\lambda_{0}$ and for any initial $\rho_{0} \neq 0$ the system will end up in the fixed point $\rho^{u p}$ (Figure 1A). If, on the contrary, the solution $\rho^{*}=0$ intersects the lower branch, this one is unstable and there is a region $\lambda_{1}<\lambda<\lambda_{0}$ for which two stable solutions $\left(\rho^{*}=0\right.$ and $\left.\rho^{u p}\right)$ coexist, separated by an unstable solution $\rho^{\text {down }}$ (Figure 1B). For $\lambda=\lambda_{1}$ the two fixed points of opposite stability annihilate through a saddlenode bifurcation, while at $\lambda=\lambda_{0}$ we still have a transcritical bifurcation. Therefore, in that region the final state of the system will be the upper branch solution $\rho^{u p}$ if the initial density $\rho_{0}>$ $\rho^{\text {down }}$ and 0 otherwise and we can observe hysteresis. For $\lambda>\lambda_{0}$ and for any initial $\rho_{0}>0$ the system will end at $\rho^{u p}$. Note that $\lambda_{0}$ is only the critical point for continuous transitions, while for discontinuous ones would be $\lambda_{1}$. The sign of the derivative of the $\rho^{*}$ function at the intersection of $\rho^{*}=0$ and the other branches determines the type of transition. If the derivative is 

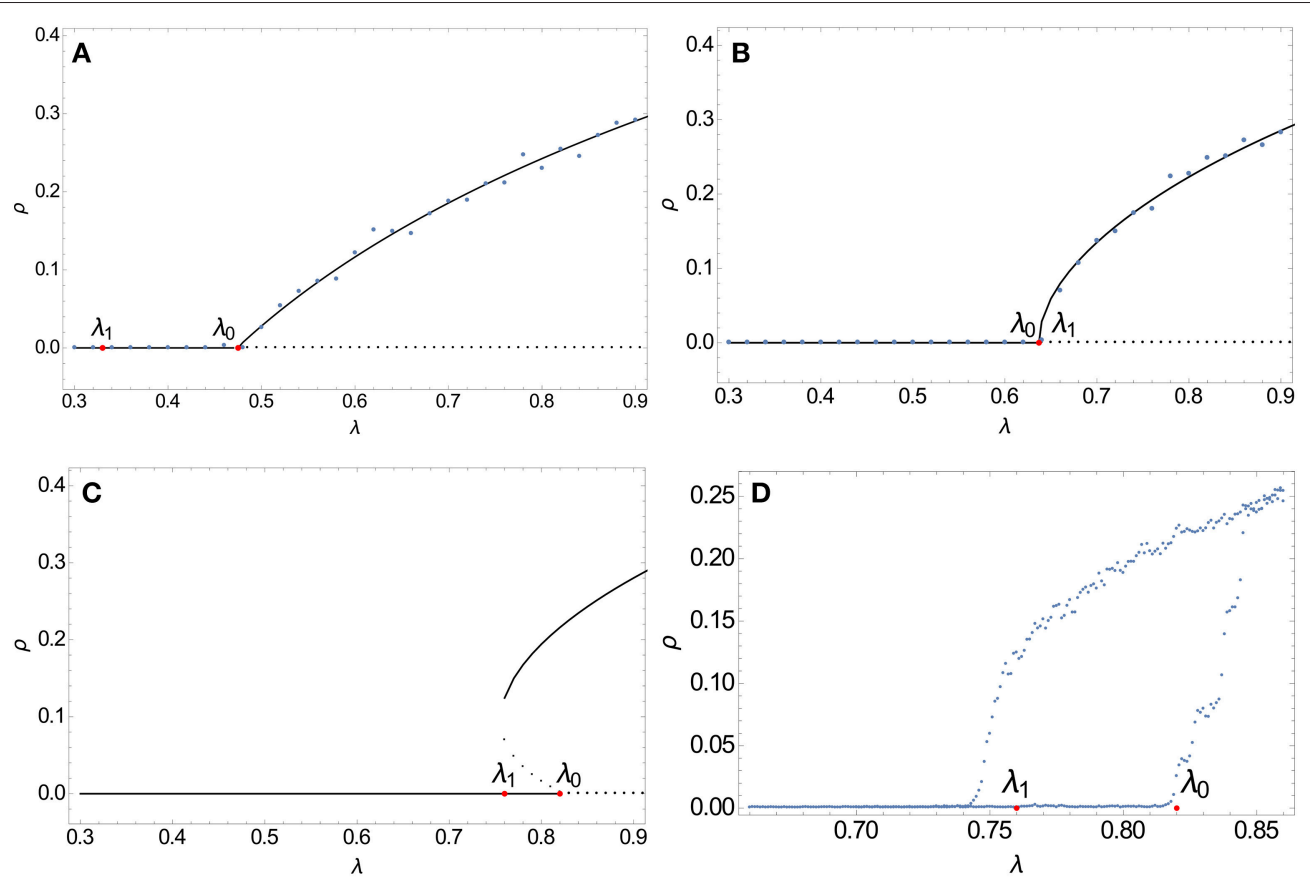

FIGURE 1 | Complete solutions of Equation (7) are shown in black for $T=3, k=10$, and $a=1.2,1.53$, 1.8 (A-C, respectively). Continuous lines represent stable solutions. Note that when $\lambda_{0}$ intersects the upper branch, the transition is continuous (A). When $\lambda_{0}$ intersects the lower branch (C), two stable solutions coexist in the region $\lambda_{1}<\lambda<\lambda_{0}, 0$ and $\rho^{u p}$, and the transition is discontinuous. Simulations of the microscopic model are shown in blue points in (A,B). For (C) the simulation is shown in (D), that amplifies the region $\lambda_{1}-\lambda_{0}$, showing the hysteresis of the system. (B) illustrates the case when $\lambda_{0}=\lambda_{1}$.

positive $\left(\rho^{*}=0\right.$ intersects $\left.\rho^{u p}\right)$, the transition is continuous, while if it is negative $\left(\rho^{*}=0\right.$ intersects $\left.\rho^{\text {down }}\right)$, the transition is discontinuous (Equations 9a,b, respectively).

$$
\begin{aligned}
& \left.\frac{d \rho^{*}}{d \lambda}\right|_{\lambda_{0}}>0 \Longrightarrow f(2)<\frac{2 k}{k-1} f(1) \\
& \left.\frac{d \rho^{*}}{d \lambda}\right|_{\lambda_{0}}<0 \Longrightarrow f(2)>\frac{2 k}{k-1} f(1) .
\end{aligned}
$$

For the particular case when $f(2)=\frac{2 k}{k-1} f(1)$ both $\lambda_{0}$ and $\lambda_{1}$ coincide. For this condition one can show, by approximating Equation (7) to third order in $\rho *$, that the bifurcation diagram is that one of a supercritical pitchfork bifurcation, i.e., the equation is equivalent to $\dot{x}=r x-x^{3}$ (Figure 1C). In this case, the final fate of the system is similar to the continuous case. For $\lambda<\lambda_{0}$ there is no global adoption and the system ends at $\rho^{*}=0$, while for $\lambda>\lambda_{0}$ any initial condition $\rho_{0} \neq 0$ will bring the system to $\rho^{u p}$.

Simulations using a microscopic model are also included in the plots of Figure 1. This microscopic model simulates an SIS dynamics in a degree regular network of $k=10$ that changes at each time step. From one step to another, an agent is selected; if it is an adopter it recovers with probability $\mu$, if not, it adopts with probability $P(n)$, where $n$ is the number of adopters among $k$ randomly chosen agents. There is an initial seed of infected agents which we fix to $1 \%$ of the total population.

In Figures 1A,B results of the simulations are shown in blue dots over the analytical solution. For Figure 1C, simulations are shown in Figure 1D. As can be seen, the system exhibits hysteresis in the region $\lambda_{1}<\lambda<\lambda_{0}$, where there is bistability. The system ends at $\rho^{u p}$ or $\rho^{\text {down }}$ depending on the initial condition.

Figure 1 also illustrates the two different kinds of transitions. The density of adopters stays at zero until a critical value of $\lambda$, where the system goes to $\rho^{u p}$ by either a continuous transition or a discontinuous transition. As can be observed, provided a value for $T$, the size of the jump increases with $a$. For values of $a \sim 1$ the system resembles the epidemic-like models while for values $a>1$ the transition is threshold-like.

For the case of our choice of $f(n)$ (Equation 2) the conditions in Equation (9) give bounds for the parameters region for which the transition is of one regime or the other:

$$
\begin{array}{ll}
\text { Cont.: } & T<\left(\frac{2^{a}(k+1)}{2^{a}(k-1)-2 k}\right)^{\frac{1}{a}} \\
\text { Disc.: } & T>\left(\frac{2^{a}(k+1)}{2^{a}(k-1)-2 k}\right)^{\frac{1}{a}} .
\end{array}
$$

Figure 2 shows this parameters space for $k=5,10,20$. The white region represents the parameters combination for a continuous transition while the light gray region corresponds to a discontinuous transition. The dark gray region is the condition that $\lambda_{0} \leq 1$ on Equation (8), that is, that the value where both curves meet is in the range $\lambda \leq 1$,

$$
T<(k-1)^{\frac{1}{a}} .
$$



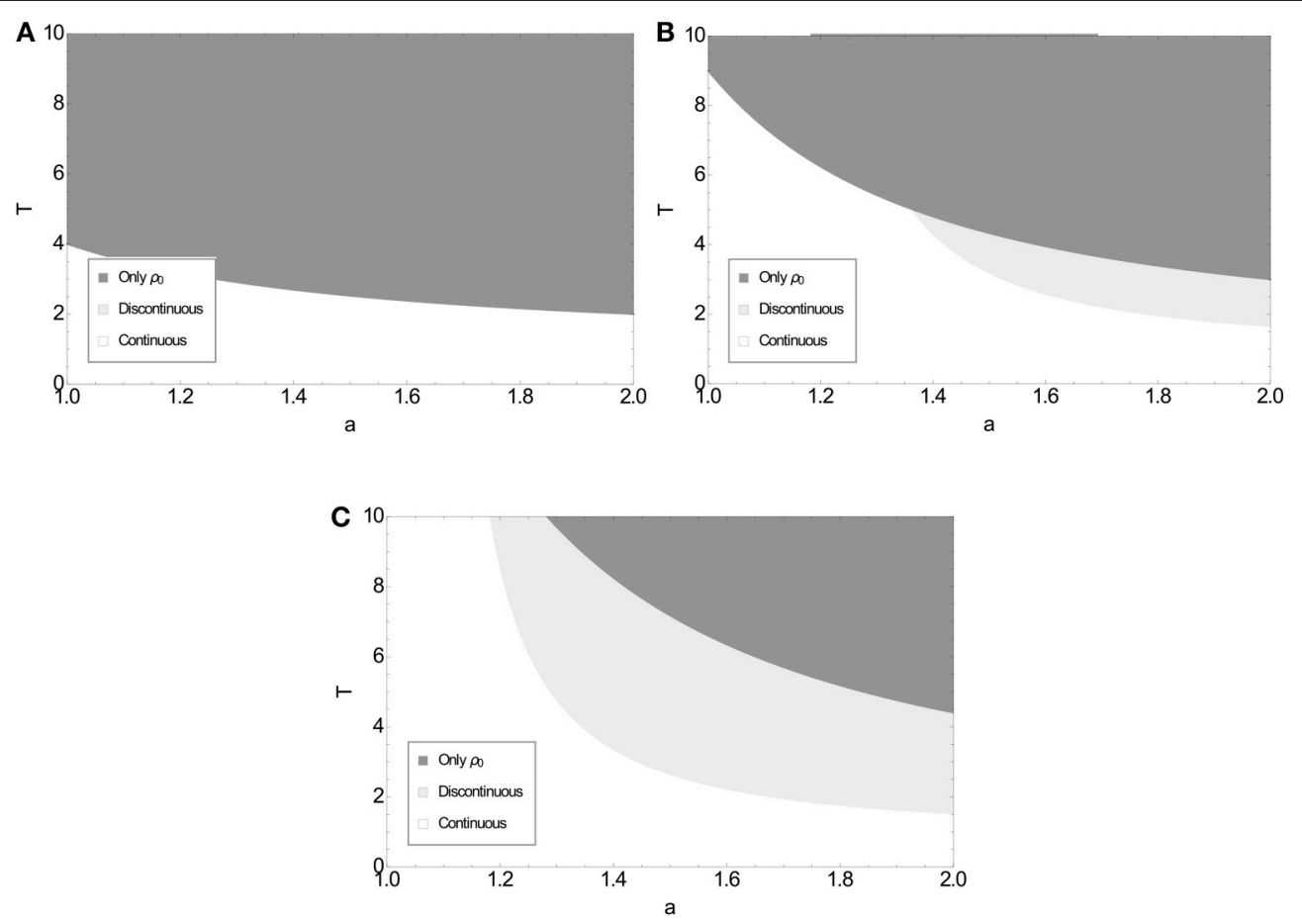

FIGURE 2 | Parameter space for a regular random network with $k=5,10,20$ (A-C, respectively). The white area is for continuous transitions while the light gray area is for discontinuous transitions. Both areas are separated by the curve given by Equation (10), corresponding to a supercritical pitchfork bifurcation diagram. In the dark gray area only the solution $\rho^{*}=0$ exists, i.e., there is not global adoption.

This constraint implies that the in dark gray region in the plot there is only one possible solution, $\rho^{*}=0$.

Both conditions together, Equations $(10,11)$, predict the values of the parameters for which the model shows one type of transition or another, or none. For example, in Figure 2B, a continuous transition is allowed for all values of $a \in[1,2]$ and some values of $T \in[0,10]$, while the discontinuous transition is only possible for values of $a$ higher than 1.25 and values of $T$ higher than 1.5. As can be seen in Figure 2, for small values of $k$, there are only continuous transitions, while for higher values of $k$, also discontinuous transitions are allowed. Besides, the higher the value of $k$, the more parameter space allows for $\rho \neq 0$ solutions.

Finally, we perform simulations to characterize numerically the behavior of the system using a similar microscopic model on quenched regular random network. Again, at each time step an agent is selected, if she is an adopter it recovers with probability $\mu$, if not, she adopts with probability $P(n)$, where now $n$ refers to the number of adopters in her network neighborhood, which is now fixed. There is an initial seed of infected agents equal to $1 \%$ of the total population. The long term values of the fraction of adopters $\rho_{\infty}$ are shown in Figure 3 for 10 realizations and different values of $a$ for $T=1.2,3$. The realizations are not averaged to show the low dispersion (inset of upper panel in Figure 3 and lower panel of Figure 3).

As Figure 2 indicates for $T=1.2$ and $k=10$, the system exhibits always a continuous transition no matter the values of $a \in[1,2]$ (inset of the upper panel). For $T=3$ and $k=10$, for values of $a$ higher than 1.5 the transition is discontinuous, as shown in Figure 2. The upper panel of Figure 3 zooms in the region of the critical point for the case of $a=1.0$. It shows the simulations of the microscopic model on a quenched degree regular random network (pink), on an annealed degree regular random network (blue) and the exact solution of the equation (black). As can be seen, there is a small discrepancy for the model on the quenched version of the network. This is because when the topology is fixed correlations appear and in particular the approximation that the infected agents are binomially distributed among the neighbors with a success probability equal to the global fraction of infected agents breaks down. As in the cases presented above, the simulations on the annealed network and the exact solution agree. For both microscopic models, the type of transition is predicted by the parameters space represented in Figure 2.

\section{CONCLUSIONS}

We have analyzed a model of social contagion (SIS-like) on degree regular random networks with an adoption probability measured in empirical data in Gallup et al. [20] that interpolates between the cases of epidemic-like spreading and thresholdlike dynamics. We show that this simple model displays both continuous and discontinuous transitions from a disease-free state to an endemic state. We find the values of the parameters that separate this transitions and the critical persuasion capacities 

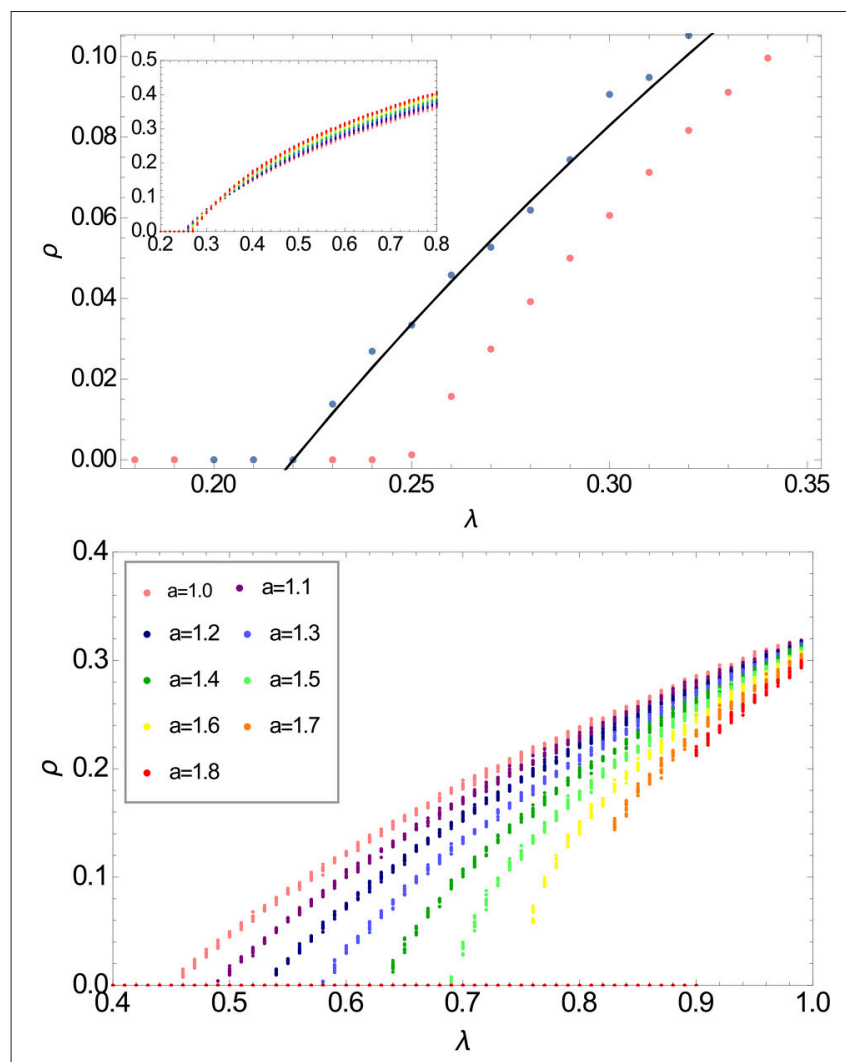

FIGURE 3 | Simulations of the microscopic model on a degree regular random network with degree $k=10$. Individuals might adopt with probability $P(n)$. Upper panel shows the results for $T=1.2$ and lower panel for $T=3$ for different values of $a$. For $T=1.2$ the transitions are continuous for any a (inset, same color code as lower panel). The upper panel shows the region of the critical point for the simulations of the microscopic model on the quenched network (pink), the simulations on the annealed network (blue) and the exact solution (black line) of the equation for $a=1.0$, respectively. For $T=3$ there are continuous or discontinuous transitions depending on the value of $a$.

$\lambda$ by applying standard linear stability and bifurcation theory tools.

The simplicity of the model studied here allows for relaxing some of the assumptions considered here. For example, the stability condition given by Equation (8) resembles the structure

\section{REFERENCES}

1. Anderson RM, May RM. Infectious Diseases of Humans: Dynamics and Control. Oxford: Oxford University Press (1991).

2. Sumpter D, Buhl J, Biro D, Couzin I. Information transfer in moving animal groups. Theory Biosci. (2008) 127:177-86. doi: 10.1007/s12064-008-0040-1

3. Rogers EM. Diffusion of Innovations. New York, NY: Simon and Schuster (1991).

4. Daley DJ, Kendall DG. Stochastic rumours. J Appl Math. (1965) 1:42-55. doi: 10.1093/imamat/1.1.42

5. González-Bailón S, Borge-Holthoefer J, Yamir M. Broadcasters and hidden influentials in online protest diffusion. Am Behav Sci. (2013) 57:943-65. doi: $10.1177 / 0002764213479371$ of the critical point in the SIS model in uncorrelated random networks with arbitrary degree distributions. Following this similarity, we conjecture that the solution of our model in complex networks will be given by $\lambda_{0}=<k>/<k^{2}>f(1)$. Thus degree heterogeneity will lead to the vanishing threshold unless $f(1) \rightarrow 0$ as $N \rightarrow \infty$. This can be achieved for example by considering that $T=c k_{m} a x$. Alternatively, an interesting variation is to consider that the adoption probability depends not on the absolute number of adopters but on the fraction of them. Besides, heterogeneity can emerge not only at the degree level, but also in the distributions of the adoption threshold $T$ and adoption coefficient $a$ and furthermore they can be correlated with the degree of the nodes. How heterogeneity affects the nature of the transition needs to be explored in detail. Another possible line of research is adding non-Markovianity to the dynamics, for example by letting the adoption probability depend not only on the state of the neighboring agents, but also on some internal time which takes into account when an agent tries to convince another one for adopting the innovation.

Our results highlight that not only the structure of the interaction network neither the dynamics alone are responsible of the type of transition that the system displays. Furthermore, this simplified framework is able to capture this seemingly disparate types of transition, which are usually taken as a signature of different dynamics. Furthermore the choice of the adoption probability curve is based on empirical measurements from Gallup et al. [20], which highlights the relevance of our results for realistic modeling of social phenomena.

\section{AUTHOR CONTRIBUTIONS}

PT, VE, and JF-G designed the research, performed the calculations, and wrote the article. PT performed the simulations.

\section{FUNDING}

JF-G and VE received funding from Agencia Estatal de Investigación (AEI) and Fondo Europeo de Desarrollo Regional (FEDER) through project SPASIMM [FIS2016-80067-P (AEI/FEDER, UE)]. Research reported in this publication was supported by research funding from King Abdullah University of Science and Technology (KAUST).

6. Alvarez R, Garcia D, Moreno Y, Schweitzer F. Sentiment cascades in the $15 \mathrm{M}$ movement. EPJ Data Sci. (2015) 4:6. doi: 10.1140/epjds/s13688-015-0042-4

7. Goel S, Watts DJ, Goldstein DG. The structure of online diffusion networks. In: Proceedings of the 13th ACM Conference on Electronic Commerce, Vol. 1. New York, NY: ACM Press (2012). p. 623-38.

8. Lopez-Pintado D, Watts DJ. Social influence, binary decisions and collective dynamics. Ration Soc. (2008) 20:399-443. doi: 10.1177/1043463108096787

9. Castellano C, Fortunato S, Loreto, V. Statistical physics of social dynamics. Rev Mod Phys. (2009) 81:591-646. doi: 10.1103/RevModPhys. 81.591

10. Pastor-Satorras R, Castellano C, Van Mieghem P, Vespignani, A. Epidemic processes in complex networks. Rev Mod Phys. (2015) 87:925-79. doi: 10.1103/RevModPhys.87.925 
11. Kermack WO, McKendrick AG. A contribution to the mathematical theory of epidemics. Proc $R$ Soc A Math Phys Eng Sci. (1927) 115:700-21. doi: 10.1098/rspa.1927.0118

12. Weiss GH, Dishon M. On the asymptotic behavior of the stochastic and deterministic models of an epidemic. Math Biosci. (1971) 11:261-5. doi: 10.1016/0025-5564(71)90087-3

13. Granovetter MS. Threshold models of collective behavior. Am J Sociol. (1978) 83:1420-43. doi: 10.1086/226707

14. Dodds PS, Watts DJ. Universal behavior in a generalized model of contagion. Phys Rev Lett. (2004) 92:218701. doi: 10.1103/PhysRevLett.92.218701

15. Bass FM. A new product growth for model consumer durables. Manage Sci. (1969) 15:215-27. doi: 10.1287/mnsc. 1040.0264

16. Young HP. Innovation diffusion in heterogeneous populations: contagion, social influence, and social learning. Am Econ Rev. (2009) 99:1899-924. doi: 10.1257/aer.99.5.1899

17. Milgram S, Bickman L, Berkowitz L. Note on the drawing power of crowds of different size. J Pers Soc Psychol. (1969) 13:79-82. doi: 10.1037/h0 028070

18. Dasgupta K, Singh R, Viswanathan B, Chakraborty D, Mukherjea S, Nanavati AA, et al. Social ties and their relevance to churn in mobile telecom networks. In: 11th International Conference on Extending Database Technology. New York, NY: ACM Press (2008).

19. Romero DM, Meeder B, Kleinberg J. Differences in the mechanics of information diffusion across topics: idioms, political hashtags, and complex contagion on twitter. In: Proceedings of the 20th international
Conference on World Wide Web. New York, NY: ACM Press (2011). p. 695-704.

20. Gallup AC, Hale JJ, Sumpter DJT, Garnier S, Kacelnik A, Krebs JR, et al. Visual attention and the acquisition of information in human crowds. Proc Natl Acad Sci USA (2012) 109:7245-50. doi: 10.1073/pnas.1116141109

21. Basios V, Nicolis S, Deneubourg J. Coordinated aggregation in complex systems. Eur Phys J Special Top. (2016) 225:1143-7. doi: 10.1140/epjst/e2016-02660-5

22. Gonze D, Abou-Jaoudé W. The goodwin model: behind the hill function. PLoS ONE (2013) 8:e69573. doi: 10.1371/journal.pone.0069573

23. Santillán M. On the use of the hill functions in mathematical models of gene regulatory networks. Math Model Nat Phenom. (2008) 3:85-97. doi: 10.1051/mmnp:2008056

Conflict of Interest Statement: The authors declare that the research was conducted in the absence of any commercial or financial relationships that could be construed as a potential conflict of interest.

Copyright (c) 2018 Tuzón, Fernández-Gracia and Eguíluz. This is an open-access article distributed under the terms of the Creative Commons Attribution License (CC $B Y)$. The use, distribution or reproduction in other forums is permitted, provided the original author(s) and the copyright owner are credited and that the original publication in this journal is cited, in accordance with accepted academic practice. No use, distribution or reproduction is permitted which does not comply with these terms. 